\title{
THE STABILITY OF BOUNDARY LAYERS ON CURVED HEATED PLATES
}

\author{
JILLIAN A. K. STOTT ${ }^{1}$ and JAMES P. DENIER ${ }^{1}$
}

(Received 23 January, 1997; revised 4 May, 2001)

\begin{abstract}
We consider the effect the competing mechanisms of buoyancy-driven acceleration (arising from heating a surface) and streamline curvature (due to curvature of a surface) have on the stability of boundary-layer flows. We confine our attention to vortex type instabilities (commonly referred to as Görtler vortices) which have been identified as one of the dominant mechanisms of instability in both centrifugally and buoyancy driven boundary layers. The particular model we consider consists of the boundary-layer flow over a heated (or cooled) curved rigid body. In the absence of buoyancy forcing the flow is centrifugally unstable to counter-rotating vortices aligned with the direction of the flow when the curvature is concave (in the fluid domain) and stable otherwise. Heating the rigid plate to a level sufficiently above the fluid's ambient (free-stream) temperature can also serve to render the flow unstable. We determine the level of heating required to render an otherwise centrifugally stable flow unstable and likewise, the level of body cooling that is required to render a centrifugally unstable flow stable.
\end{abstract}

\section{Introduction}

Our concern is with the interplay of buoyancy and centrifugal forces in promoting, or inhibiting, the growth of longitudinal vortices in boundary-layer flows over curved heated surfaces. We consider this problem in the context of both linear and nonlinear vortex instabilities.

The competition between buoyancy-driven and curvature (or centrifugally) induced instabilities has received little attention although their driving mechanisms arise in a wide variety of physically important fluid flows. These competing instabilities can arise in two different ways. For example, in compact heat exchangers the design aim is to maximise the efficiency of the apparatus in removing heat from a surface while keeping the dimensions of the device to a minimum. Because of the limitations on

\footnotetext{
${ }^{1}$ Department of Applied Mathematics, The University of Adelaide, Adelaide SA 5005, Australia.

(C) Australian Mathematical Society 2002, Serial-fee code 1446-8735/02
} 
size, body curvature is invariably encountered; one typical application of curvature is in the form of channel corrugations [9, 22] (see also the review article [17]). The effect of such body curvature upon the buoyancy induced instabilities (and any subsequent transition to turbulence) is then an important design issue when viewed from the standpoint of maximising efficiency through turbulent heat transfer. On the other hand, in cases where centrifugal instabilities naturally arise due to surface concavity, such as on the underside of a laminar-flow control airfoil [18], the question of whether wall cooling can be used to control the instability is an important one.

Typically in applications involving heat transfer a natural asymptotic limit arises based upon the fact that the Grashof number is large. The Grashof number $G r$ is defined as

$$
G r=L^{3} g \beta \Delta T / v^{2},
$$

where $L$ is a characteristic length scale of the flow, $g$ is the acceleration due to gravity, $\beta$ the coefficient of thermal expansion, $v$ the kinematic viscosity of the fluid, and $\Delta T$ the temperature difference between the heated surface of interest and the ambient fluid temperature. For water at $20 \mathrm{degC}$ the kinematic viscosity is $v=1.004 \times 10^{-2} \mathrm{~cm}^{2} / \mathrm{sec}$ and the coefficient of thermal expansion $\beta=2.1 \times 10^{-4} \mathrm{deg}^{-1} \mathrm{C}$ (see Batchelor [2, pp. 594-597]). If we consider a device in which the characteristic length scale $L$ is equal to $10 \mathrm{~cm}$ and the temperature difference $\Delta T$ is $20 \mathrm{degC}$ then the Grashof number is $O\left(10^{9}\right)$. If we replace water with air the Grashof number is slightly reduced to $O\left(10^{8}\right)$. In both cases we can confidently refer to the flow as having a large Grashof number.

Such flows (that is, flows over heat surfaces) typically exhibit instabilities in the form of spanwise periodic, counter-rotating vortices which are aligned with the direction of the flow; one such example can be found in the work of Goldstein and Sparrow [9]. There are a number of studies that consider the asymptotic, large Grashof number, form of disturbances to boundary layer flows over heated flat plates. They have identified two distinct régimes for the span-wise wavelength of longitudinal vortices which appear to dominate the flow development. Hall and Morris [15] examined the linear stability of a forced-convection boundary layer over a horizontal flat plate. They demonstrated that such a flow is indeed unstable to longitudinal vortices which are aligned with the direction of the flow. For $O(1)$ values of the Grashof number the instabilities have wavelength that are of order unity (relative to the boundary layer thickness) and develop in a non-parallel manner. It is however, only in the limit of large Grashof number $G r \gg 1$ that the fastest growing mode (on the basis of linear theory) is present. This mode is governed by a quasi-parallel theory and has a growth rate of $O\left(G r^{3 / 4}\right)$, a span-wise wavelength of $O\left(G r^{-1 / 4}\right)$ and is confined to within a viscous sub-layer of thickness $O\left(\mathrm{Gr}^{-1 / 4}\right)$ situated at the surface of the heated plate. Because the disturbance is localized near the wall surface, surface inhomogeneities can readily 
induce this form of instability. Furthermore Hall and Morris [15] demonstrated that in the limit of small vortex wavenumber the vortex mode takes on the characteristics of an oblique Tollmien-Schlichting wave which is progressively destabilised by wall heating.

The work of Hall and Morris [15] is closely connected to the earlier work of Timoshin [21] and Denier, Hall and Seddougui [5] who, independently, identified the most unstable Görtler vortex mode in the boundary-layer flow over a concave wall. This mode is confined to an $O\left(G^{-1 / 5}\right)$ thickness wall bounded shear layer, has a span-wise wavelength of $O\left(G^{-1 / 5}\right)$ and evolves in the stream-wise direction with a growth-rate of $O\left(G^{3 / 5}\right)$, where $G \gg 1$ is the Görtler number of the flow. One significant difference that arises between the results of [15] and [5] is that in the latter study the most unstable mode lies in a wavenumber régime distinct from that which contains the right-hand branch of the curve of neutral stability. However, in both cases the most unstable mode is confined to a viscous sub-layer close to the plate.

Hall and Lakin [14] extended the earlier work of Hall [11], concerning the nonparallel evolution of weakly nonlinear short wavelength vortices, into a strongly nonlinear régime. In this strongly nonlinear régime the flow is governed by a first harmonic/mean-flow interaction in which the vortex induced mean flow is of the same order of magnitude as the underlying basic flow (and not a small correction to the mean flow as would arise in a traditional Stuart-Landau type of weakly nonlinear stability theory). The vortices exist across a finite part of the boundary layer (away from the wall) and their existence causes the stream-wise mean flow component to be radically altered from that encountered in the absence of the vortices. Similar results were found by Hall [12] in his investigation of the nonlinear stability of mixed forced-free convection boundary layers over horizontal flat plates. However, in that case it was shown that the vortex structure is confined to that part of the boundary layer adjacent to the heated plate. Under certain conditions the mean flow component is modified to the extent that points of inflection develop and the flow becomes susceptible to rapidly growing high frequency Rayleigh (or Taylor-Goldstein) wave modes. This result is in stark contrast with situations where the vortex is not present, in which case the boundary-layer flow is stable to such wave-like disturbances. It is the presence of the strongly nonlinear vortices which renders the flow susceptible to rapidly growing secondary instabilities.

It is the aim of the present paper to consider various aspects of the stability of buoyant boundary layers to longitudinal vortex motions and the effect concave/convex curvature has on such instabilities. As a first step towards an increased understanding of such flows we restrict our attention to steady vortex (that is, span-wise periodic) modes of instability. The question of wave-like disturbances in buoyant boundarylayer flows has recently been considered by Mureithi, Denier and Stott [20], Mureithi and Denier [19] and Denier and Mureithi [6] and for the case of buoyant Poiseuille 
flow by Denier and Stott [7]. The first two of the aforementioned papers were predominantly concerned with the modifications to the stability characteristics of Tollmien-Schlichting waves. The latter papers, however, were concerned with the nonlinear evolution of a single monochromatic short wavelength travelling wave. The evolution of such a wave mode was shown to be governed by a wave/mean-flow interaction theory remarkably similar to that found for short wavelength vortex modes by Hall and Lakin [14]. These wave motions invariably persist in the presence of underlying body curvature (both concave and convex) and as such have implications to the nonlinear vortex modes to be described here. The question as to which mode, wave or vortex, dominates the flow is a question of some importance from the perspective of predicting transition to turbulence. A definitive answer to this question is outside the scope of this paper.

In isolation, the results discussed above present a somewhat confusing picture in that there are a large number of asymptotic régimes which appear to be disconnected. To put these results into context we must refer to the question of the receptivity of the flow to longitudinal vortices, that is, the question of how external disturbances become internalized in the flow and how these develop into vortex-like motions. This question has been considered in the context of curvature induced [5] and buoyancy induced vortices [15]. Hall and Morris [15] demonstrated that, in high Grashof number flows, disturbances in the free-stream (such as incipient turbulence) are not as efficient in generating the fastest growing vortex as are disturbances localized near (or on) the surface (such as isolated 'hot spots'). They (that is, free-stream disturbances) can however serve to excite short wavelength vortices that are localized within the body of the boundary layer. On the other hand, disturbances with spanwise wavelengths of order unity (with respect to the boundary layer thickness) will tend to promote vortices whose spanwise wavelength is also $O(1)$. We therefore have a picture where the response of the boundary layer in promoting vortices is intimately linked to the form of the environmental disturbance present in the flow.

This paper therefore presents a systematic study of the competing effects of buoyancy and centrifugal forces on vortex instabilities across the whole (vortex) wavenumber spectrum. To do this we first formulate the equations that describe the flow of a mixed forced-free convection boundary layer over a curved heated plate in Section 2. In Section 3 we examine the linear stability of such flows in the natural limit of large Grashof number. We first consider inviscid disturbances which arise when $G r \gg 1$ and the vortex wavelength is of the same order as the boundary layer thickness. The effect of concave/convex curvature on the inviscid vortex modes is investigated. Still within the context of a linearized instability theory, the effect of concave or convex curvature on the most unstable (or fastest growing) vortex mode is then considered. In Section 4 we turn our attention to the effect of nonlinearity on the vortex instability. With the Grashof number $G r \gg 1$ the evolution of short wavelength vortices 
is governed by the vortex/mean-flow interaction theory of Hall and Lakin [14]. The effect of the competing buoyancy and centrifugal forces on finite amplitude vortices is investigated. Finally in Section 5 we draw some conclusions from our work and suggest some directions for future work.

\section{Formulation}

Consider the flow of a viscous fluid over a heated wall of variable curvature and let $L$ denote a typical length-scale over which the curvature changes. If $U_{\infty}$ is a typical speed a large distance from the wall, and $v$ is the kinematic viscosity, we define the Reynolds number, $R e$, as

$$
R e=U_{\infty} L / \nu .
$$

We initially take the temperature of the plate to be uniform and equal to $T_{0}$, although a variable temperature along the plate facilitates the development of a similarity solution of the boundary-layer equations to be considered in subsequent sections, and assume that sufficiently far from the plate the temperature of the flow is $T_{\infty}$. The location of the bounding wall is defined with respect to dimensional Cartesian coordinates $x^{*}, y^{*}$, $z^{*}$ by

$$
y^{*}=L \operatorname{Re}^{-1 / 2} \hat{g}\left(x^{*} / L\right) .
$$

We define non-dimensional coordinates

$$
(x, \hat{y}, z)=\left(x^{*}, \operatorname{Re}^{1 / 2} y^{*}, \operatorname{Re}^{1 / 2} z^{*}\right) / L,
$$

and take the corresponding dimensionless velocity field to be

$$
(u, \hat{v}, w)=\left(u^{*}, R e^{1 / 2} v^{*}, R e^{1 / 2} w^{*}\right) / U_{\infty},
$$

whilst the dimensionless temperature is given by

$$
T^{*} / T_{\infty}=1+\left(T_{0} / T_{\infty}-1\right) T .
$$

If we let $\bar{\rho}$ denote a typical fluid density then we may write the dimensionless pressure as

$$
p=\frac{p^{*}}{\bar{\rho} U_{0}^{2}}=\left(\bar{p}_{0}(x)+\frac{\bar{p}_{1}(x)}{R e^{1 / 2}}+\frac{G r}{R e^{5 / 2}}(\bar{p}(x, \hat{y})+p(x, \hat{y}, z))+\cdots\right),
$$

where the Grashof number $G r$ is defined in (1) with $\Delta T=T_{0}-T_{\infty}$. The dimensionless fluid density is given by

$$
\rho=\rho^{*} / \bar{\rho}=1+\beta\left(T^{*}-T_{\infty}\right)
$$


where $\beta$ is the coefficient of thermal expansion of the fluid.

Substituting the above dimensionless variables into the Navier-Stokes equations, taking the limit $\operatorname{Re} \rightarrow \infty$ and employing the Boussinesq approximation we obtain

$$
\begin{aligned}
& \quad \bar{u}_{x}+\bar{v}_{y}+\tilde{U}_{x}+\tilde{V}_{y}+\tilde{W}_{z}=0, \\
& -\bar{u} \bar{u}_{x}-\bar{v} \bar{u}_{y}+u_{e} u_{e x}+(1 / 2) S \bar{p}_{y} G \Psi(x)-S \bar{p}_{x}+\bar{u}_{y y}+\tilde{U}_{y y}+\tilde{U}_{z z} \\
& \quad=\bar{u} \tilde{U}_{x}+\tilde{U}_{x}+\bar{v} \tilde{U}_{y}+\tilde{V}_{y}+Q_{1}, \\
& -\bar{p}_{y}+\bar{\theta}-\tilde{P}_{y}+G r \tilde{\Theta}-G \chi \tilde{U} \bar{u}+\tilde{V}_{y y}+\tilde{V}_{z z}=\bar{u} \tilde{V}_{x}+\tilde{U} \bar{v}_{x}+\bar{v} \tilde{V}_{y}+\tilde{V} \bar{v}_{y}+Q_{2}, \\
& \quad \tilde{P}_{z}+\tilde{W}_{y y}+\tilde{W}_{z z}=\bar{u} \tilde{W}_{x}+\bar{v} \tilde{W}_{y}+Q_{3}, \\
& -\bar{u} \bar{\theta}_{x}-\bar{v} \bar{\theta}_{y}+\left(\bar{\theta}_{y y}+\tilde{\Theta}_{y y}+\tilde{\Theta}_{z z}\right) / \sigma=\bar{u} \tilde{\Theta}_{x}+\tilde{U} \bar{\theta}_{x}+\bar{v} \tilde{\Theta}_{y}+\tilde{V} \bar{\theta}_{y}+Q_{4},
\end{aligned}
$$

which must be solved subject to the boundary conditions

$$
\begin{aligned}
\bar{u}=\bar{v}=\bar{\theta}-1=0 & \text { on } y=0, \\
\tilde{U}=\tilde{V}=\tilde{W}=\tilde{\Theta}=0 & \text { on } y=0, \\
\bar{u} \rightarrow u_{e}(x), \quad \bar{\theta} \rightarrow 0 & \text { as } y \rightarrow \infty, \\
(\tilde{U}, \tilde{V}, \tilde{W}, \tilde{\Theta}) \rightarrow 0 & \text { as } y \rightarrow \infty .
\end{aligned}
$$

In deriving (2)-(3) we have written

$$
(u, v, w, p, T)=(\bar{u}, \bar{v}, \bar{w}, \bar{p}, \bar{\theta})+(\tilde{U}, \tilde{V}, \tilde{W}, \tilde{P}, \tilde{\Theta}),
$$

so that the total flow field is decomposed into a basic boundary layer flow (denoted by barred quantities) and a superimposed disturbance. We have also made use of the Prandtl transformation $\hat{y}=y+\hat{g}(x), \hat{v}=v+\hat{g}(x) u$ to transform the boundary conditions on the surface $\hat{y}=\hat{g}(x)$ to boundary conditions on $y=0$. In (3c) we have introduced the term

$$
G \chi=2 \hat{g}^{\prime \prime}(x),
$$

which arises as a result of the Prandtl transformation. Here $G$ and $\chi$ are commonly referred to as the Görtler number and the wall curvature respectively. We have defined $G \Psi(x)=2 \hat{g}^{\prime}(x)$ and $\bar{p}_{0 x}=-u_{e} u_{e x}$. The parameter $S=G r / R e$ is the buoyancy parameter introduced by Hall [12], $\sigma$ is the Prandtl number and $Q_{j}$ contains the nonlinear terms:

$$
\begin{array}{ll}
Q_{1}=\tilde{U} \tilde{U}_{x}+\tilde{V} \tilde{U}_{y}+\tilde{W} \tilde{U}_{z}, & Q_{2}=\tilde{U} \tilde{V}_{x}+\tilde{V} \tilde{V}_{y}+\tilde{W} \tilde{V}_{z}+G \chi \tilde{U}^{2} / 2, \\
Q_{3}=\tilde{U} \tilde{W}_{x}+\tilde{V} \tilde{W}_{y}+\tilde{W} \tilde{W}_{z}, & Q_{4}=\tilde{U} \tilde{\Theta}_{x}+\tilde{V} \tilde{\Theta}_{y}+\tilde{W} \tilde{\Theta}_{z} .
\end{array}
$$

The precise details of this formulation are well documented in previous studies such as [5] and [15]. 


\section{The growth of linear instabilities in the boundary layer}

We shall initially confine our attention to small amplitude disturbances to the basic boundary-layer flow. Consider then the Fourier decomposition of the disturbance quantities in the span-wise direction

$$
(\tilde{U}, \tilde{V}, \tilde{W}, \tilde{P}, \tilde{\Theta})=\delta(\tilde{u}, \tilde{v}, \tilde{w}, \tilde{p}, \tilde{\theta}) e^{i k z}+O\left(\delta^{2}\right)
$$

where $\delta$ is an infinitesimally small quantity and $2 \pi / k$ is the wavelength of the (linear) vortex with amplitudes $\tilde{u}, \tilde{v}$ etc. Substituting into (2) and equating coefficients of powers of the vortex amplitude $\delta$ to zero gives the equations governing the unperturbed boundary layer:

$$
\begin{aligned}
\bar{u}_{x}+\bar{v}_{y} & =0, \\
\bar{p}_{y} & =\bar{\theta}, \\
\bar{u} \bar{u}_{x}+\bar{v} \bar{u}_{y} & =\bar{u}_{y y}+u_{e} u_{e x}-S \bar{p}_{x}+(1 / 2) S \bar{\theta} G \Psi(x), \\
\bar{u} \bar{\theta}_{x}+\bar{v} \bar{\theta}_{y} & =\bar{\theta}_{y y} / \sigma,
\end{aligned}
$$

with corresponding boundary conditions

$$
\bar{u}=\bar{v}=\bar{\theta}-1=0 \quad \text { on } y=0, \quad \bar{u} \rightarrow u_{e}, \quad \bar{\theta} \rightarrow 0 \quad \text { as } y \rightarrow \infty .
$$

The linearized equations governing the disturbance amplitude are obtained at $O(\delta)$ as

$$
\begin{aligned}
\tilde{u}_{x}+\tilde{v}_{y}+i k \tilde{w} & =0 \\
\bar{u} \tilde{u}_{x}+\tilde{u} \bar{u}_{x}+\bar{v} \tilde{u}_{y}+\tilde{v} \bar{u}_{y} & =\tilde{u}_{y y}-k^{2} \tilde{u} \\
\bar{u} \tilde{v}_{x}+\tilde{u} \bar{v}_{x}+\bar{v} \tilde{v}_{y}+\tilde{v} \bar{v}_{y}+G \chi(x) \bar{u} \tilde{u} & =-\tilde{p}_{y}+G r \tilde{\theta}+\tilde{v}_{y y}-k^{2} \tilde{v} \\
\bar{u} \tilde{w}_{x}+\bar{v} \tilde{w}_{y} & =-i k \tilde{p}+\tilde{w}_{y y}-k^{2} \tilde{w} \\
\bar{u} \tilde{\theta}_{x}+\tilde{u} \bar{\theta}_{x}+\bar{v} \tilde{\theta}_{y}+\tilde{v} \bar{\theta}_{y} & =\left(\tilde{\theta}_{y y}-k^{2} \tilde{\theta}\right) / \sigma
\end{aligned}
$$

with corresponding boundary conditions

$$
\tilde{u}=\tilde{v}=\tilde{w}=\tilde{\theta}=0 \quad \text { on } y=0, \quad(\tilde{u}, \tilde{v}, \tilde{w}) \rightarrow 0 \quad \text { as } y \rightarrow \infty .
$$

By cross-differentiating (5c) and (5d) we can eliminate the pressure to give

$$
\begin{aligned}
\left(k^{2} \bar{v}_{x}\right. & \left.+k^{2} G \chi \bar{u}+\bar{u}_{x x y}\right) \tilde{u}+\left(k^{2} \bar{v}_{y}+\bar{u}_{x y y}+k^{4}\right) \tilde{v} \\
& +2\left(\bar{u}_{x y}+\bar{u}_{x} \frac{\partial}{\partial y}\right) \tilde{u}_{x}+\left(k^{2} \bar{u}+\bar{u}_{y y}-\bar{u} \frac{\partial^{2}}{\partial y^{2}}\right) \tilde{v}_{x}-\left(2 k^{2}+\bar{v}_{y}\right) \tilde{v}_{y y} \\
& +\left(k^{2} \bar{v}+\bar{u}_{x y}\right) \tilde{v}_{y}+\bar{v} \tilde{u}_{y y}+\tilde{v}_{y y y y}-\bar{v} \tilde{v}_{y y y}-k^{2} G r \tilde{\theta}=0,
\end{aligned}
$$


to be solved subject to

$$
\tilde{u}=\tilde{v}=\tilde{v}_{y}=\tilde{\theta}=0 \quad \text { on } y=0, \infty .
$$

With the basic velocity and temperature fields given by (4), the non-parallel evolution of a single vortex mode can be determined by solving (5b), (5e) and (6) numerically; $\tilde{w}$ and $\tilde{p}$ can then be determined from (5a) and (5d) respectively. Such a numerical solution requires the imposition of a disturbance at some initial stream-wise location. The parabolic (in $x$ ) partial differential equations can then be marched forward in $x$ and the growth or decay of the disturbance can be monitored by, for example, the kinetic energy of the disturbance; the reader is referred to Hall [11] for details of this marching procedure. We do not pursue a purely numerical solution of the disturbance equations here since our primary concern is with the large Grashof number limit typically encountered in buoyant boundary-layer flows (see the discussion in Section 1). In this large Grashof number limit considerable analytic progress can be made towards a solution of (6).

Before proceeding with a discussion of the large Grashof number limit we note the presence of the buoyancy parameter $S$ in the stream-wise momentum equation (4b). The first term $S \bar{p}_{x}$ is a buoyancy induced acceleration which is present in both curved and flat plate buoyant boundary layers. The second term $S G \Psi \bar{\theta} / 2$ is also a buoyancy induced acceleration but arises due to the curvature of the underlying plate; in the case of a concavely curved body, for which $G \chi>0$, this term has a retarding influence on the boundary layer in regions where $G \Psi<0$ (that is, $\hat{g}^{\prime}<0$ ) and acts to accelerate the flow in regions where $G \Psi>0$ (that is, $\hat{g}^{\prime}>0$ ). The converse holds in the case of a boundary-layer flow over a convexly curved surface.

In order to highlight the effect of buoyancy coupling and the streamline curvature of the basic flow on the stability properties of the flow we shall consider the cases $S=0$ and $S$ non-zero separately. First, let us focus on the case $S=0$.

\subsection{Inviscid vortex modes in the large Grashof number limit Turning our} attention to the large Grashof number limit, with $S$ set equal to zero, we first neglect the effects of viscosity on those vortex modes with $O(1)$ span-wise wavelengths. This assumption is made on the basis of the results of Hall and Morris [15] who found that it is only at asymptotically large and infinitesimally small vortex wavelengths that the effects of viscosity are felt by the disturbance. Thus, if we allow $G r \gg 1$ and take the span-wise wavenumber $k$ to be $O(1)$ (corresponding to the inviscid vortex modes) then, in order to achieve a balance between stream-wise advection, normal diffusion and buoyancy, we must have $\partial_{x} \sim G r^{1 / 2}$ and $\tilde{v}=O\left(G r^{1 / 2} \tilde{u}\right)$. Thus we write

$$
\left(\tilde{u},|G r|^{-1 / 2} \tilde{v}, \tilde{\theta}\right)=\left(u_{0}, v_{0}, \theta_{0}\right) \exp \left(|G r|^{1 / 2} \int \hat{\beta}(x) d x\right),
$$


where $u_{0}, v_{0}$ and $\theta_{0}$ are functions of $x$ and $y$ only and $\hat{\beta}$ expands as

$$
\hat{\beta}=\beta_{0}+\beta_{1}|G r|^{-1 / 2}+\cdots .
$$

We also note that the centrifugal acceleration of the flow induced by the underlying body curvature first exerts an influence on the inviscid vortex modes when the Görtler number is of the same order of magnitude as the Grashof number, that is,

$$
G=G_{0}|G r|+\cdots .
$$

Substituting these expansions into (6), we obtain to leading order, upon eliminating $u_{0}$ and $\theta_{0}$,

$$
\frac{\partial^{2} v_{0}}{\partial y^{2}}-\frac{1}{\bar{u} \beta_{0}}\left(\left(k^{2} \bar{u}+\bar{u}_{y y}\right) \beta_{0}+\operatorname{sign}(G r) \frac{k^{2} \bar{\theta}_{y}}{\bar{u} \beta_{0}}-\frac{k^{2} G_{0} \chi \bar{u}_{y}}{\beta_{0}}\right) v_{0}=0 .
$$

This equation must be solved subject to the usual inviscid boundary conditions $v_{0}=0$ on $y=0$ and $y \rightarrow \infty$ and defines an eigenvalue problem for $\beta_{0}=\beta_{0}(k)$. Equation (7) is a modified version of the Taylor-Goldstein equation; the reader is referred to Drazin and Reid [8] for a discussion of its relevance to the stability of stratified shear flows and to Blackaby and Choudhari [3] for a discussion of its relevance to the stability properties of weakly three-dimensional boundary layers.

Equation (7) is readily solved using a second order accurate finite-difference scheme in which the homogeneous boundary condition $v_{0}=0$ on $y=0$ is replaced by $v_{0 y}=1$ on $y=0$; Newton iteration is then performed on $\beta_{0}$ until the remaining boundary condition $v_{0}=0$ on $y=0$ is satisfied (to within some desired tolerance). A useful check on the accuracy and validity of our numerical scheme is obtained by comparison with the exact solution of (7), identified by Denier et al. [5] and Timoshin [21], which exists when $\bar{\theta}_{y}=0$ and, in the absence of curvature $G_{0}=0$, with the results of Hall and Morris [15]. Our numerical scheme is able to reproduce both these limiting cases.

For simplicity, the basic flow is taken to be of the Falkner-Skan type given by

$$
\bar{u}=x^{1 / 3} f^{\prime}(\eta), \quad \bar{v}=\frac{\eta f^{\prime}(\eta)-2 f(\eta)}{3 x^{1 / 3}}, \quad \bar{\theta}=x^{1 / 3} g(\eta), \quad \bar{p}=x^{2 / 3} q(\eta),
$$

where the similarity variable $\eta=y / x^{1 / 3}$. Although other choices of similarity variable are available, the $x^{1 / 3}$ form was chosen so as to provide a point of comparison with the earlier work of Hall and co-workers [12, 15]. The functions $f, g$ and $q$ in (8) are determined from the solution of the system of equations

$$
\frac{1}{3} f^{\prime 2}-\frac{2}{3} f f^{\prime \prime}=f^{\prime \prime \prime}+\frac{1}{3}, \quad q^{\prime}=g, \quad \frac{1}{\sigma} g^{\prime \prime}=\frac{1}{3} f^{\prime} g-\frac{2}{3} g^{\prime}
$$


with boundary conditions

$$
f=f^{\prime}=g-1=0 \quad \text { on } \eta=0, \quad f^{\prime}-1, g, q \rightarrow 0 \quad \text { as } \eta \rightarrow 0 .
$$

In order for a similarity solution to exist we must take a curvature profile $\chi=x^{-1 / 3} \chi_{1}$. Note also that as we are currently concerned with the case of $S=0$ the buoyancy induced acceleration terms are absent from (9a).

The results of our calculation are presented in Figure 1, for the first eigen-mode, in the form of plots of the growth rate $\beta_{0}$ as a function of the wavenumber for a variety of curvature profiles. These results are for a Prandtl number equal to unity (that is, $\sigma=1$ in (9)); changing the value of $\sigma$ has only a quantitative effect on these results and henceforth we will set $\sigma=1$ in all calculations in this section.

From Figure 1 we observe that if the plate is curved in a concave manner, corresponding to $\chi_{1} G_{0}>0$, the flow permits disturbances with larger growth rates than if the plate were flat and the growth rates increase with increased curvature. Linear inviscid vortex disturbances persist when the plate is curved in a convex manner, and hence in this parameter régime the destabilising effect of the unstable temperature stratification outweighs the stabilising influence of convex curvature. The stabilizing effect of the underlying convex body curvature increases as the local radius of curvature of the body is decreased. Indeed physical intuition leads us to the conclusion that, given any level of buoyancy, centrifugal forces will ultimately stabilize the flow if the radius of curvature of a convexly curved body is suitably small ${ }^{1}$. Indeed, in the case $G_{0} \gg 1$, the growth rate $\beta_{0}$ appearing in (7) should be re-scaled as $\beta_{0}=G_{0}^{1 / 2} \hat{\beta}_{0}$ (with all other quantities unchanged). In the limit $G_{0} \rightarrow \infty$ the resulting equation is identical to that derived by Denier et al. [5, (5.8)] and thus the growth rate $\hat{\beta}_{0}^{2}=\chi k / 2$. Thus in the case $\chi<0$ (that is, in the case of a convexly curved surface) the inviscid modes are ultimately re-stabilized.

We also note that when the Grashof number was negative no unstable modes could be located at moderate values of $G_{0}$. However, as noted above, in the limit $G_{0} \gg 1$ centrifugal forces will again dominate with (9) reducing to the linear inviscid vortex equation (see [5] and [21] for details). That equation possesses a full spectrum of unstable solutions if $\chi>0$ and so on this basis we can state that for a given value of $G r<0$ (corresponding to the case of a cooled rigid wall) there will be a critical value of $G_{0}$ beyond which the flow will be unstable to inviscid longitudinal vortex motions.

We now turn our attention to the case of strong buoyancy coupling within the basic boundary-layer flow, corresponding to $S$ non-zero. The effect of the underlying body curvature is now felt when the Görtler number is an $O(1)$ quantity. In this case the curvature effect arises not through the equations governing the disturbance but through

\footnotetext{
${ }^{1}$ There is, of course, the proviso that the radius of curvature of the body be of such a size that the assumption of an attached boundary-layer flow is valid.
} 


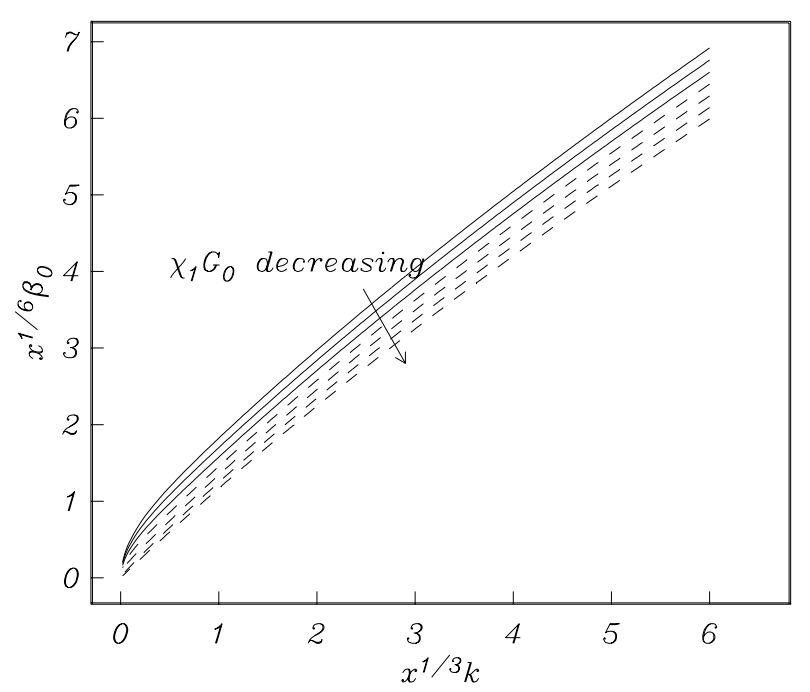

FIGURE 1. Growth rates (for $\sigma=1$ ) of an inviscid vortex mode as a function of the span-wise wavenumber $k$ with scaled Görtler numbers $\chi_{1} G_{0}=3,2,1,0,-1,-2,-3$ and $\operatorname{sign}(G r)=1$. The dashed curves correspond to $\chi_{1} G_{0} \leq 0$.

the modification to the underlying boundary-layer flow as a result of the combined effect of centrifugal acceleration and buoyancy. Thus with the growth rate defined as before, the disturbance equation, first derived by Hall and Morris [15], is given by

$$
\frac{\partial^{2} v_{0}}{\partial y^{2}}-\frac{1}{\bar{u} \beta_{0}}\left(\left(k^{2} \bar{u}+\bar{u}_{y y}\right) \beta_{0}+\operatorname{sign}(G r) \frac{k^{2} \bar{\theta}_{y}}{\bar{u} \beta_{0}}\right) v_{0}=0,
$$

(which is equivalent to (7) with $G_{0} \chi$ formally set equal to zero). The self-similar form for the boundary-layer flow is still appropriate, however, the stream-wise momentum equation is now given by

$$
\frac{1}{3} f^{\prime 2}-\frac{2}{3} f f^{\prime \prime}=f^{\prime \prime \prime}+\frac{1}{3}-\frac{2}{3} S q+\frac{1}{3} S \eta q^{\prime}+\frac{1}{2} S G_{1} g
$$

which in the limit $S \rightarrow 0$ reduces to the stream-wise momentum equation of (9). Note that in order to develop a similarity solution it was necessary to choose $G \Psi(x)=$ $G_{1} x^{-2 / 3}$ where $G_{1}$ is a constant and that the $x^{-2 / 3}$ factor in $G \Psi$ signifies that the body is convex, whereas the sign of $G_{1}$ is determined by whether $\hat{g}^{\prime}$ is positive or negative. The case of $G_{1}$ positive corresponds to $\hat{g}^{\prime}>0$. Such a convention is employed in order to allow us to make use of a similarity solution. Non self-similar solutions must be considered by solving the full system of partial differential equations.

The precise value, and sign, of $G_{1}$ depends upon the form of the bounding surface. Indeed $G \Psi=2 \hat{g}^{\prime}$ where $\hat{g}$ denotes the position of the rigid surface. As such $G_{1}$ is 


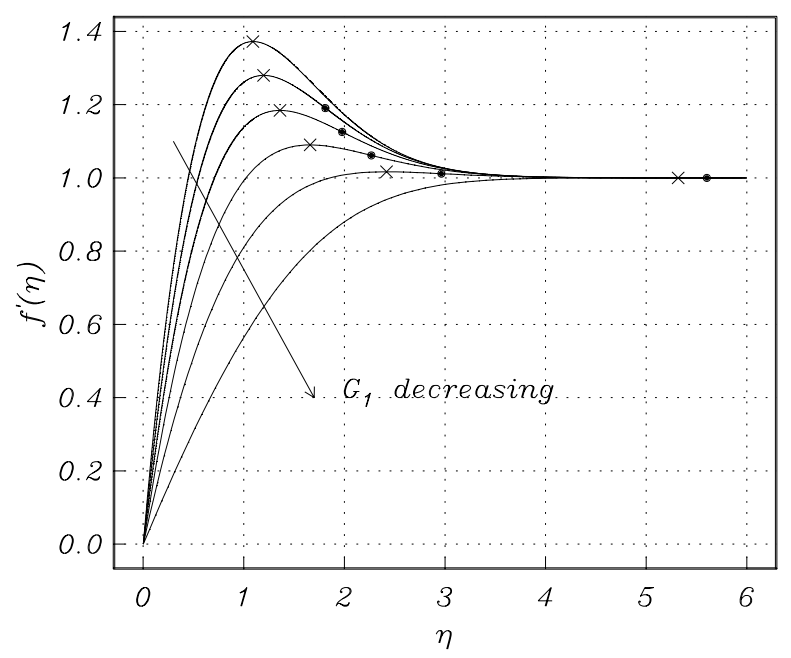

FIGURE 2. The stream-wise velocity of the basic flow for $\sigma=1, S=1$ and $G_{1}=-2,0,2,4,6,8$. Here $\times$ denotes stationary points and $\bullet$ denotes inflection points.

related to the gradient of the rigid surface and can take both positive or negative values (irrespective of whether the surface has concave or convex curvature). The effect of the curvature is now felt, not through a centrifugal acceleration in the disturbance equations, but through the variable slope of the body and acts as an additional buoyancy induced acceleration/deceleration on the stream-wise component of the basic flow.

In Figure 2 we present a plot of the stream-wise velocity component of the flow for a number of values of $G_{1}$; included in this figure are the stationary points (that is, local maxima of $f^{\prime}$ ). The effect of buoyancy induced acceleration is readily observed; such an acceleration causes the stream-wise velocity to 'overshoot' its free-stream value. The effect of decreasing $G_{1}$ on the basic flow is (a) to decrease the local maximum and move the inflexion point associated with this local maximum to successively higher values of $\eta$ and (b) for increasingly negative values of $G_{1}$ a new inflexion point emerges from $\eta=0$ and ultimately the similarity solution breaks down when $G_{1}$ reaches a level at which $f^{\prime \prime}=0$; the similarity solution is no longer valid and the flow cannot be described under the assumption of an attached boundary layer. A plot of disturbance growth rates, determined from (10), is shown in Figure 3 for the representative cases $S=0.5$ and $S=2.0$. The effects of the 'curvature' induced acceleration are readily seen; the growth rates for the decelerated boundary layer, with $G_{1}<0$, are significantly higher than those for the case $G_{1}=0$ (corresponding to a flat plate). The above situation is reversed for the boundary-layer flow over a concave body. Thus although the effect of curvature is not felt in the disturbance equation it has a considerable effect on the growth rates of inviscid vortex modes. 


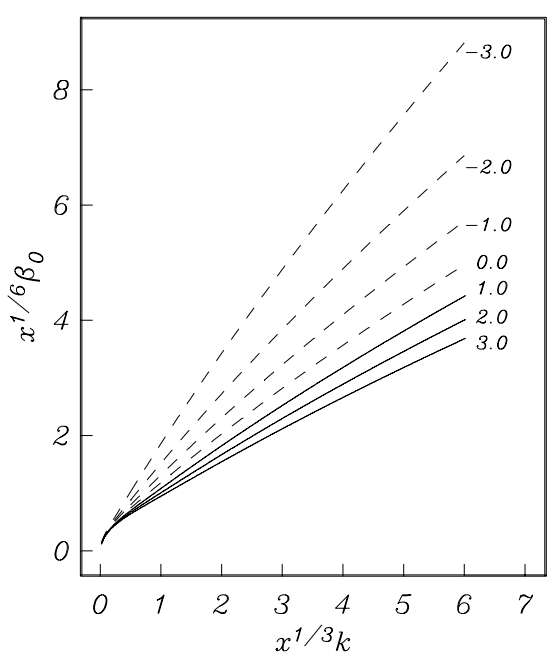

(a)

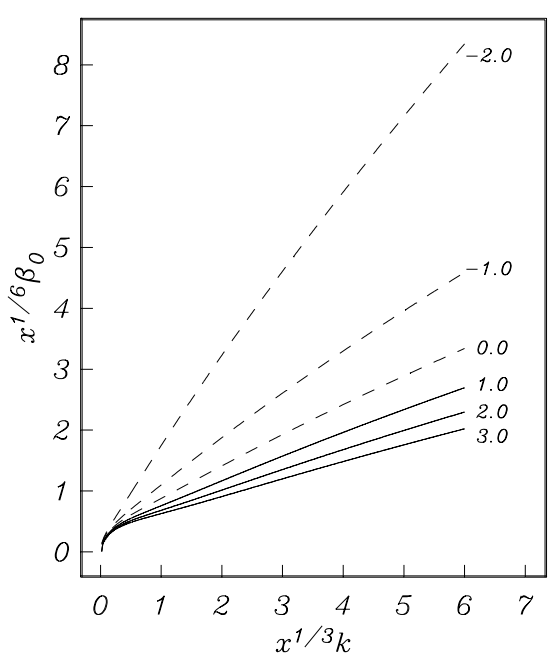

(b)

FiguRE 3. Growth rate curves for (a) $S=0.5$, (b) $S=2.0$, where for each figure $G_{1}=-3,-2,-1,0$, $1,2,3$ respectively and $\sigma=1$.

3.2. The most unstable vortex in the large Grashof number limit The results of the previous section focused on vortices with wavenumber $k=O(1)$. As noted in Section 1, this mode of instability will generally be induced by environmental disturbances that have a similar $O(1)$ spanwise periodicity. However, environmental disturbances (such as small-scale isolated 'hot spots') introduced through the bounding surface will produce a response in the flow that is (initially) localized near the surface and having a spanwise wavelength that is short compared to the boundary layer thickness $[5,15]$.

The short wavelength limit of (10) is amenable to analysis. Such an analysis has been previously carried out by Denier et al. [5] and Hall and Morris [15] (the reader is referred to those papers for full details). The outcome of a large wavenumber analysis of the inviscid vortex equation is the identification of the fastest growing wall bounded mode. We will now turn our attention to the most unstable mode in order to determine the effect of body curvature on this mode.

As shown by Hall and Morris [15], the fastest growing vortex within a buoyant boundary layer is confined to a wall-bounded shear layer of thickness $O\left(\mathrm{Gr}^{-1 / 4}\right)$, has a span-wise wavelength of $O\left(\mathrm{Gr}^{-1 / 4}\right)$ (the vortex retaining an aspect ratio of one) and has a stream-wise growth rate of $O\left(G r^{3 / 4}\right)$. In order to elucidate the structure of this mode we therefore write

$$
k=|G r|^{1 / 4} k_{0}, \quad \zeta=k y,
$$


and expand the disturbance quantities as

$$
\left(\tilde{u},|G r|^{-1 / 2} \tilde{v}, \tilde{\theta}\right)=\left[\left(u_{0}, v_{0}, \theta_{0}\right)+O\left(|G r|^{-1 / 4}\right)\right] \exp \int|G r|^{3 / 4} \hat{\beta}(x) d x,
$$

where $u_{0}, v_{0}, \theta_{0}$ are functions of $\zeta$ only and the scaled growth rate $\hat{\beta}=\beta_{0}+|G r|^{1 / 4} \beta_{1}+$ .... An a posteori estimate shows that the underlying body curvature first affects the most unstable vortex mode when

$$
G=\tilde{G}_{0}|G r|^{5 / 4} .
$$

Substituting the above expansions into the linear disturbance equations (6) yields, to leading order in inverse powers of $|G r|^{1 / 4}$,

$$
\begin{aligned}
\mathscr{L} u_{0} & =\mu v_{0} / k_{0}^{2}, \\
\mathscr{L}\left(\frac{\partial^{2}}{\partial \zeta^{2}}-1\right) v_{0} & =\operatorname{sign}(G r) \frac{\theta_{0}}{k_{0}^{2}}-\frac{\tilde{G}_{0} \chi \mu \zeta u_{0}}{k_{0}^{3}}, \\
\mathscr{L}_{1} \theta_{0} & =-v_{0} \tau / k_{0}^{2}
\end{aligned}
$$

to be solved subject to the boundary conditions

$$
u_{0}=v_{0}=v_{0}^{\prime}=\theta_{0}=0, \quad \zeta=0, \infty
$$

and where the differential operators appearing in (12) are defined as

$$
\mathscr{L}=\left(\frac{\partial^{2}}{\partial \zeta^{2}}-1-\frac{\zeta \mu \beta_{0}}{k_{0}^{3}}\right), \quad \mathscr{L}_{1}=\left(\frac{1}{\sigma} \frac{\partial^{2}}{\partial \zeta^{2}}-\frac{1}{\sigma}-\frac{\zeta \mu \beta_{0}}{k_{0}^{3}}\right) .
$$

In deriving (12) the basic flow has been expanded in the form

$$
\bar{u}=\mu \zeta k^{-1}+\cdots, \quad \bar{\theta}=1-\tau \zeta k^{-1}+\cdots .
$$

By re-scaling

$$
\begin{aligned}
& \beta_{00}=\left(\mu \tau^{-3 / 4}\right) \beta_{0}, \quad k_{00}=\tau^{-1 / 4} k_{0}, \quad G_{00}=\chi \mu^{2} \tilde{G}_{0} / \tau^{5 / 4}, \\
& u_{00}=\tau^{1 / 2} u_{0} / \mu, \quad v_{00}=v_{0}, \quad \theta_{00}=\theta_{0} / \tau^{1 / 2},
\end{aligned}
$$

(12) can be written in canonical form

$$
\begin{aligned}
\mathscr{L}^{+} u_{00} & =v_{00} / k_{00}^{2} \\
\mathscr{L}^{+}\left(\frac{\partial^{2}}{\partial \zeta^{2}}-1\right) v_{00} & =\operatorname{sign}(G r) \frac{\theta_{00}}{k_{00}^{2}}-\frac{\operatorname{sign}(\chi) G_{00} \zeta u_{00}}{k_{00}^{3}}, \\
\mathscr{L}_{1}^{+} \theta_{00} & =-v_{00} / k_{00}^{2}
\end{aligned}
$$




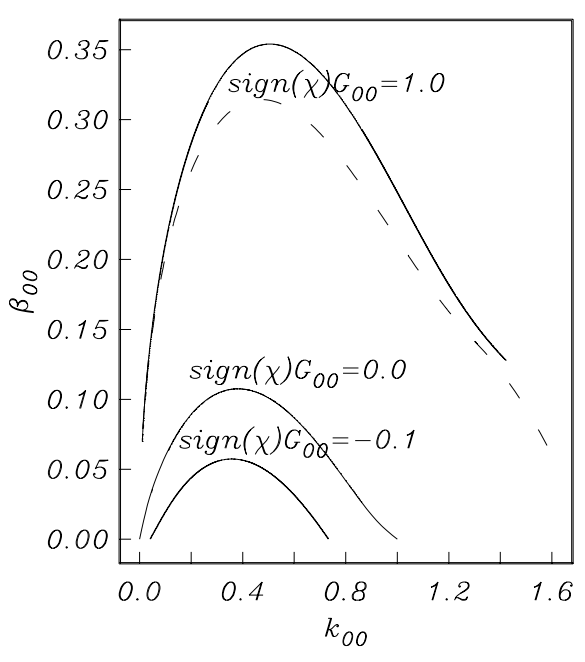

(a)

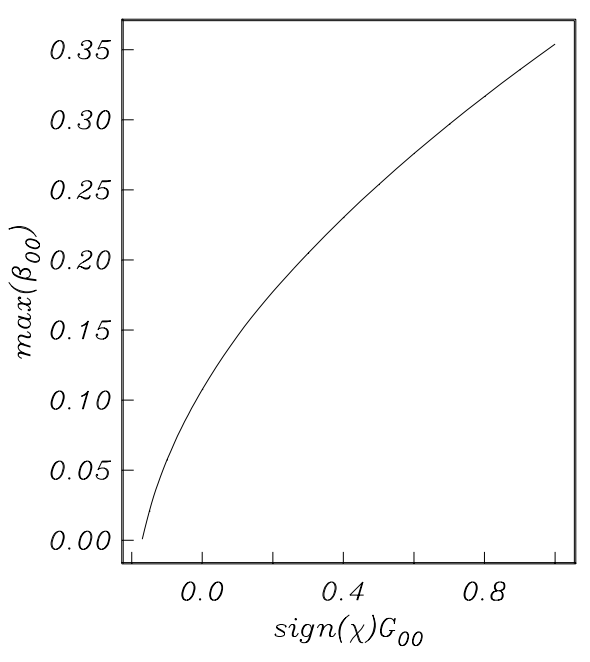

(b)

FIGURE 4. (a) Linear, viscous growth rates of the most unstable mode for various curvatures as a function of the wave-speed for the case $\sigma=1$. The dashed line corresponds to $\operatorname{sign}(\chi) G_{00}=1$ in (13b); (b) The maximum value of the linear, viscous growth rate as a function of $\operatorname{sign}(\chi) G_{00}$.

with boundary conditions

$$
u_{00}=v_{00}=v_{00}^{\prime}=\theta_{00}=0, \quad \zeta=0, \infty .
$$

Here $\mathscr{L}^{+}, \mathscr{L}_{1}^{+}$are $\mathscr{L}, \mathscr{L}_{1}$ transformed by the variables described above.

Note that the buoyancy parameter $S$ does not explicitly appear in system (13), and the results to be presented below for the scaled growth rate $\beta_{00}$ do not depend upon $S$. However, it is important to note that both the skin friction $\mu$ and the temperature gradient at the surface $-\tau$ do depend implicitly upon $S$ (see system (4)).

The eighth order system (13) defines an eigenvalue problem for the growth-rate $\beta_{00}$ as a function of the scaled wavenumber $k_{00}$. This system was solved by replacing the homogeneous boundary condition $u_{00}=0$ on $\zeta=0$ by an inhomogeneous boundary condition $u_{00}^{\prime}=1$ on $\zeta=0$. The resulting two-point boundary-value problem was then solved using the routine D02RAF from the NAG suite of subroutines. Newton iteration was performed on $\beta_{00}$ until the homogeneous boundary condition, $u_{00}=0$ on $\zeta=0$, was satisfied to within some desired tolerance.

The results of several such calculations are presented in Figure 4. Figure 4 (a) presents plots of the growth rate versus wavenumber for an unstably stratified boundary layer (corresponding to $G r \geq 0$ ). The dashed curve corresponds to the $G r$ term set equal to zero in (13), in which case the disturbance temperature and momentum fields de-couple and the results of Denier et al. [5] are recovered. The middle curve, for 
which $G_{00}=0$, reproduces the results of Hall and Morris [15] (both cases provide a valuable validation of the numerical scheme used in the present paper). We note from the $G_{00}=0$ curve that the neutral vortex mode is found at wavenumber $k_{00}=1$ and therefore the $k=O\left(G r^{1 / 4}\right)$ wavenumber régime contains the right-hand-branch of the neutral curve (as noted in [15]). The results presented in this figure demonstrate, perhaps not surprisingly, that concave curvature has a de-stabilizing influence on the flow whereas convex curvature has a stabilizing influence. In Figure 4 (b) we present a plot of the maximum growth rate as a function of the scaled curvature parameter $G_{00}$. Here we observe that the maximum growth rate tends to zero as $\chi G_{00}$ becomes progressively more negative. Thus for large levels of convex curvature $\left(\chi G_{00}<0\right)$ the most unstable vortex mode is ultimately re-stabilized. It is a consequence of the fact that the most unstable mode is confined to a thin viscous wall layer that leads to this result that a high degree of convex curvature, of $O\left(G r^{5 / 4}\right)$, is required to fully stabilize the flow. Thus, for the flow of a highly heated boundary layer over a body curved in a convex fashion, the flow remains susceptible to longitudinal-type vortex motions for a wide range of curvature profiles.

\section{The nonlinear saturation of short wavelength vortices}

We now turn our attention to the question of the nonlinear saturation of the vortex. Generally this is a question that can only be resolved by resorting to a full numerical solution of the full nonlinear system (2). However, in the limit of large Grashof number, this system can be solved asymptotically to give information on the nonlinear response of the flow to a finite amplitude vortex. In effect, a weakly nonlinear theory arises which is based upon the fact that a neutrally stable vortex has a wavelength that is small compared to the boundary layer thickness (see [11]). With this fact in mind, we now turn our attention to the effect of buoyancy forcing on the nonlinear, centrifugally driven, vortex instability.

In the absence of buoyancy forcing, the results of Hall and Lakin [14] demonstrate that the nonlinear vortex motion is confined to a region of depth $O(1)$ located a finite distance from the bounding surface. Thus, following the formulation in [14], we suppose that there exists a vortex of wavelength $k$, confined to a region of depth $O(1)$, some distance $y=y_{1}(x)$ away from the plate. The vortex activity is confined to a region between $y_{1}(x)$ and $y_{2}(x)$, with passive viscous transition regions centred on $y_{1}$ and $y_{2}$ of thickness $k^{-2 / 3}$ which are required to smooth the decay of the vortices (see [14] for full details). A consequence of these passive viscous layers is that all the basic flow quantities $\bar{u}, \bar{v}, \bar{q}, \bar{T}$ (and their first derivatives) are continuous at $y_{1}(x)$ and $y_{2}(x)$. In the following analysis it is assumed that

$$
G r=G r_{0} k^{4}+\cdots
$$


and $S=G r / R e$ is an $O(1)$ quantity.

We have already shown in the linear analysis of Section 3 that there are significant differences in the stability characteristics and disturbance structure of flows in which $S$ is allowed to formally approach zero and flows in which $S$ is non-zero. We anticipate similar differences in the nonlinear analysis by considering the two cases separately.

4.1. No buoyancy coupling Consider the case when the effects of buoyancy coupling are negligible, that is, when $S \rightarrow 0$. We follow the methodology of [14] by first expanding the Görtler number as

$$
G=k^{4} G_{0}+\cdots
$$

The basic flow expands as

$$
(\bar{u}, \bar{v}, \bar{p}, \bar{\theta})=\left(\bar{u}_{0}, \bar{v}_{0}, \bar{p}_{0}, \bar{\theta}_{0}\right)+k^{-2 / 3}\left(\bar{u}_{1}, \bar{v}_{1}, \bar{p}_{1}, \bar{\theta}_{1}\right)+\cdots
$$

whereas the perturbation quantities expand as

$$
(\tilde{U}, \tilde{V}, \tilde{W}, \tilde{P}, \tilde{\Theta})=k^{-1}\left[\left(U_{0}, k^{2} V_{0}, k W_{0}, k^{2} P_{0}, \Theta_{0}\right) E+c . c\right]+\cdots .
$$

Here $2 E=e^{i k z}$ and $c . c$ denotes the complex conjugate of the preceding term. We emphasize here that the mean flow, given by (16), is that which is induced by the small amplitude vortex motion, represented by (17).

By substituting (15)-(17) into (2) and equating firstly the leading order mean terms and then the leading order fundamental terms, the fully coupled vortex amplitude/mean flow field equations can be written as

$$
\begin{aligned}
\bar{u}_{0 x}+\bar{v}_{0 y} & =0, \quad \bar{q}_{0 y}=\bar{\theta}_{0}, \\
\bar{u}_{0} \bar{u}_{0 x}+\bar{v}_{0} \bar{u}_{0 y}-\bar{u}_{0 y y} & =u_{e} u_{e x}-\frac{1}{2} H\left(V_{0}^{2}\right) \frac{\partial}{\partial y}\left(\bar{u}_{0 y} V_{0}^{2}\right), \\
\bar{u}_{0} \bar{\theta}_{0 x}+\bar{v}_{0} \bar{\theta}_{0 y}-\frac{1}{\sigma} \bar{\theta}_{0 y y} & =\frac{1}{2} H\left(V_{0}^{2}\right) \frac{\partial}{\partial y}\left(\sigma \bar{\theta}_{0 y} V_{0}^{2}\right)
\end{aligned}
$$

and

$$
H\left(V_{0}^{2}\right)\left(\bar{\theta}_{0 y}-\frac{G_{0} \chi \bar{u}_{0} \bar{u}_{0 y}}{G r_{0} \sigma}+\frac{1}{G r_{0} \sigma}\right)=0,
$$

where $H$ is the Heaviside function defined by

$$
H(s)= \begin{cases}1 & \text { if } s>0 \\ 0 & \text { if } s \leq 0\end{cases}
$$

The boundary conditions for this system are

$$
\bar{u}_{0}=\bar{v}_{0}=\bar{\theta}_{0}-\mathscr{T}_{0}=0, \quad \text { on } y=0,
$$




$$
\bar{u}_{0} \rightarrow u_{e}, \quad \bar{\theta}_{0} \rightarrow 0 \quad \text { as } y \rightarrow \infty .
$$

Thus the problem becomes the solution of the system (18a)-(19) subject to (20). The system of equations (18a) and (19) requires some explanation. Outside the region of vortex activity, where $V_{0} \equiv 0$, the system (18a) reduces to the standard boundary-layer equations. Within the region of vortex activity the Heaviside function in (18a)-(19) is set equal to unity; the resulting equations are a system of five partial differential equations in five dependent variables $V_{0}, \bar{u}_{0}, \bar{v}_{0}, \bar{\theta}_{0}$ and $\bar{q}_{0}$. The outer limits of the region of vortex activity, $y_{1}(x)$ and $y_{2}(x)$ at which $V_{0}=0$, are to be determined as part of the solution process. At $y_{1}$ and $y_{2}$ we have the additional requirement that $\bar{u}_{0}, \bar{\theta}_{0}$, $\bar{v}_{0}$ and $\bar{q}_{0}$ (and their first derivatives) are continuous. It is possible to solve (18a)-(19) using a marching procedure in the stream-wise direction; see Hall and Lakin [14] and Denier and Hall [4] for details. We shall, however, restrict our attention to a self-similar solution to this problem. This simplification allows us to concentrate on the interaction between the buoyancy and centrifugal forcing at play in the problem. We therefore focus our efforts on the Falkner-Skan profile defined in Section 3 for which the basic flow variables are

$$
\begin{array}{llll}
\bar{u}_{0}=x^{1 / 3} f^{\prime}(\eta), & \bar{v}_{0}=\frac{\eta f^{\prime}(\eta)-2 f(\eta)}{3 x^{1 / 3}}, & \bar{\theta}_{0}=x^{1 / 3} g(\eta), & V_{0}=V(\eta), \\
\chi=x^{-1 / 3} \chi_{1}, & \bar{q}_{0}=x^{2 / 3} q(\eta), & u_{e}=x^{1 / 3}, & \mathscr{T}_{0}=x^{1 / 3} .
\end{array}
$$

Note that this similarity form was adopted by Hall [12] thus allowing for direct comparison with his results; it also serves as a useful check on our numerical results. Using this similarity solution (18a)-(20) become

$$
\begin{aligned}
f^{\prime \prime \prime}+\left(2 f^{\prime \prime} f-f^{\prime 2}+1\right) / 3 & =-H\left(V^{2}\right)\left(f^{\prime \prime} V^{2}\right)^{\prime} / 2, \\
q^{\prime} & =g, \\
g^{\prime \prime} / \sigma-\left(f^{\prime} g-2 f g^{\prime}\right) / 3 & =-\sigma H\left(V^{2}\right)\left(g^{\prime} V^{2}\right)^{\prime} / 2
\end{aligned}
$$

and

$$
H\left(V^{2}\right)\left(g^{\prime}-\frac{G_{0} \chi_{1}}{G r_{0} \sigma} f^{\prime} f^{\prime \prime}+\frac{1}{G r_{0} \sigma}\right)=0,
$$

with boundary conditions

$$
\begin{aligned}
& f=f^{\prime}=g-1=0, \quad \text { on } \eta=0, \\
& f^{\prime}-1=g=q=0, \quad \text { as } \quad \eta \rightarrow \infty .
\end{aligned}
$$

In addition to the boundary conditions (24) we require the continuity of the variables $f, f^{\prime}, f^{\prime \prime}, g, g^{\prime}$ and $q$ at $\eta=\eta_{1}, \eta_{2}$ where $V=0$. We solve (22a)-(24) by making initial guesses for $f^{\prime \prime}(0), g^{\prime}(0)$ and $q(0)$ and integrating (22a) from $\eta=0$ to some value of $\eta=\eta_{1}^{*}$ where (23) is satisfied. Equations (22a)-(23) are then integrated 
TABLE 1. The critical values of the scaled Görtler number $G_{0}$ and critical position for Prandtl numbers $\sigma=0.5$ and $\sigma=1.5$. Note that in the case $G r_{0}=0$ the energy and momentum fields decouple thus giving results which are independent of the Prandtl number.

\begin{tabular}{|c|c|c||c|c|c|}
\hline \multicolumn{3}{|c||}{$\sigma=0.5$} & \multicolumn{3}{c|}{$\sigma=1.5$} \\
\hline \hline$G r_{0}$ & $\eta$ & $G_{0}$ & $G r_{0}$ & $\eta$ & $G_{0}$ \\
\hline 0.0 & 1.0045 & 3.9252 & 0.0 & 1.0045 & 3.9252 \\
\hline 1.0 & 0.9295 & 3.2680 & 0.25 & 0.9495 & 3.2453 \\
\hline 2.0 & 0.8346 & 2.5393 & 0.50 & 0.8695 & 2.4255 \\
\hline 3.0 & 0.7046 & 1.7631 & 0.75 & 0.7492 & 1.6314 \\
\hline 4.0 & 0.5047 & 0.9653 & 1.00 & 0.4597 & 0.5969 \\
\hline 4.77 & 0.0899 & 0.1046 & 1.08 & 0.0299 & 0.0216 \\
\hline
\end{tabular}

from $\eta_{1}^{*}$, with initial conditions obtained from the previous region and the additional condition that $V\left(\eta_{1}^{*}\right)=0$. The integration continues until $\eta=\eta_{2}^{*}$ where $V$ is again zero (to within a specified tolerance). Equation (22a) is integrated from $\eta=\eta_{2}^{*}$ to some suitably large value of $\eta=\eta_{\infty}$. At this stage we will not in general satisfy the boundary conditions at infinity and therefore Newton iteration is used to update the initial guesses for $f^{\prime \prime}(0), g^{\prime}(0)$ and $q(0)$ until the far field boundary conditions are satisfied to within some desired tolerance. All integrations were performed using a fourth order Runge-Kutta quadrature scheme.

The results obtained from the numerical procedure described above may be found in Figure 5 where we present plots of the upper and lower limits of the region of vortex activity as a function of the scaled Görtler number for a variety of scaled Grashof numbers. We note firstly that the $G r_{0}=0$ curve corresponds to the case of purely centrifugal forcing considered by Hall and Lakin [14] (note however that [14] considered a Blasius boundary layer, and not the Falkner-Skan basic flow profile under consideration here). For a fixed value of $G r_{0}$ there is a critical value of the Görtler number below which the flow is linearly stable to longitudinal counter rotating vortices. (Note that it becomes increasingly difficult to obtain convergence of our numerical scheme as the critical Görtler number is approached and as such the critical Görtler number could not be calculated.) However, it is possible to calculate the critical Görtler number (and its critical location) using the method presented in Denier et al. [5]. The results of this calculation are presented in Table 1, for two different Prandtl numbers $\sigma=0.5$ and $\sigma=1.5$.

From the results presented in Figure 5 and Table 1 we note that as the buoyancy forcing is increased, corresponding to increasing $G r_{0}$, the region of vortex activity is extended and the critical Görtler number is decreased. By further increasing $G r_{0}$ a level at which the critical Görtler number is zero can be attained; this limit is, however, singular (in the context of our numerical scheme) since the lower limit of 
vortex activity now becomes $y_{1}(x) \equiv 0$. The flow structure in this limit (with $G_{0}$ formally set equal to zero) has been described by Hall [12] and will be considered further in Section 4.2 below.

In Figure 6 we present plots of the vortex induced stream-wise velocity $f^{\prime}$ and the vortex amplitude $V_{0}$ for the case of $G_{0}=4.0$ and a variety of values of $G r_{0}$. The effect of increasing $G r_{0}$ is readily seen. The region of vortex activity expands and the vortex amplitude is increased. In addition the vortex induced mean flow moves further from that found in the unperturbed mean flow and ultimately develops points of inflexion. The flow will then be susceptible to secondary instabilities (see references [13] and [16] for details).

4.2. The case when the buoyancy parameter $S$ is non-zero When $S$ is non-zero the linear analysis of Section 3 demonstrated that the effect of curvature is felt first in the basic flow; this is also the case when the instabilities are strongly nonlinear. The situation when $S$ is non-zero is similar to the findings of Hall [12] where it was shown that small wavelength vortices feel the locally destabilising effect of the vertical temperature gradient. This gradient reaches a maximum at the plate and hence it is in this region that the instability is initiated. Thus we assume that the vortex is situated close to the plate and allow $y_{1}(x) \rightarrow 0$. The flow is first modified by curvature effects when the Görtler number is $O(1)$. The basic flow variables expand as before and the equations for the flow field are

$$
\begin{aligned}
\bar{u}_{0 x}+\bar{v}_{0 y} & =0 \\
\bar{q}_{0 y} & =\bar{\theta}_{0}, \\
\bar{u}_{0} \bar{u}_{0 x}+\bar{v}_{0} \bar{u}_{0 y}-\bar{u}_{0 y y} & =u_{e} u_{e x}-S \bar{q}_{0 x}+\frac{S}{2} G \bar{\theta}_{0} \Psi-\frac{H\left(V_{0}^{2}\right)}{2} \frac{\partial}{\partial y}\left(\bar{u}_{0 y} V_{0}^{2}\right), \\
\bar{u}_{0} \bar{\theta}_{0 x}+\bar{v}_{0} \bar{\theta}_{0 y}-\frac{1}{\sigma} \bar{\theta}_{0 y y} & =\frac{H\left(V_{0}^{2}\right)}{2} \frac{\partial}{\partial y}\left(\sigma \bar{\theta}_{0 y} V_{0}^{2}\right)
\end{aligned}
$$

and

$$
H\left(V_{0}^{2}\right)\left(\bar{\theta}_{0 y}+\frac{1}{G r_{0} \sigma}\right)=0,
$$

where the boundary conditions are given by (20) with the minor modification that the region of vortex activity extends from $y=0$ to $y=y_{2}(x)$. In this case the value of the vortex amplitude $V_{0}(x, 0)$ at the wall is an unknown and must be determined as part of the solution process. Because of the non-vanishing of the vortex amplitude at the boundary a viscous wall layer is required, situated at $y=0$, in which the total flow adjusts so as to satisfy the full no-slip boundary conditions; this layer is passive and we omit a description of it here.

We again use the Falkner-Skan similarity solution to describe the basic flow vari- 


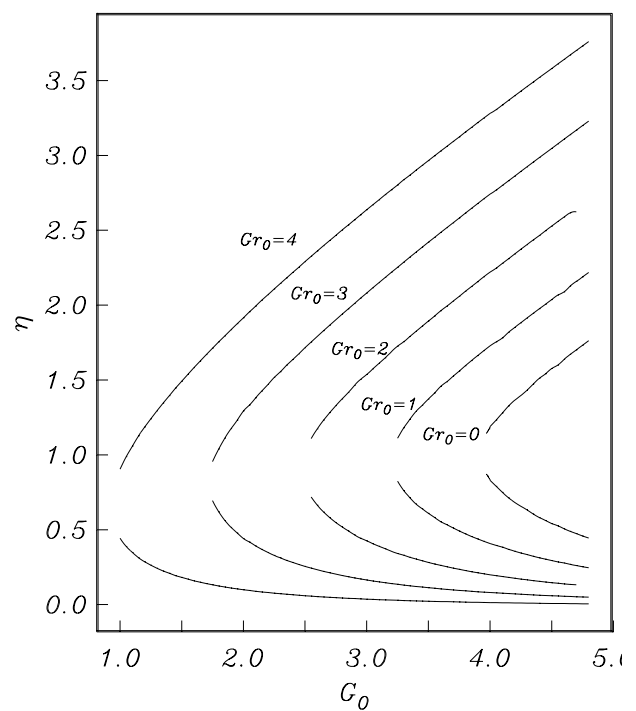

FIGURE 5. The region of existence of the vortex as a function of curvature at $G r_{0}=0.0,1.0,2.0,3.0,4.0$ for $\sigma=0.5$.

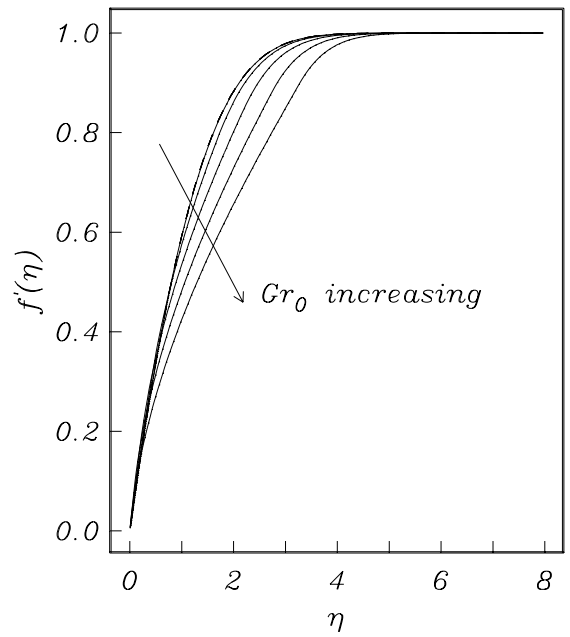

(a)

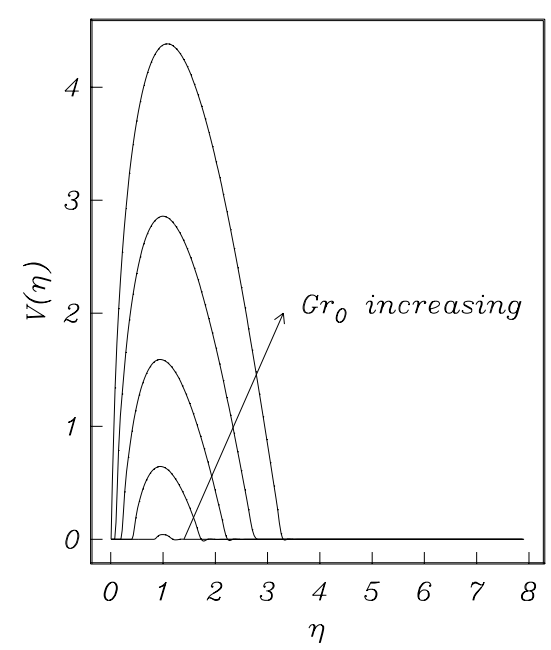

(b)

FIGURE 6. An eigenfunction plot for (a) $f^{\prime}(\eta)$, (b) $V\left(\eta\right.$ ) for $\sigma=0.5, G_{0}=4.0$ and $G r_{0}=0,1,2,3,4$ with $\chi_{1}=1$. 
ables, see (21), with $G \Psi=G_{1} x^{-2 / 3}$. Equations (25a) and (26) then become

$$
\begin{aligned}
f^{\prime \prime \prime}+\frac{1}{3}\left(2 f^{\prime \prime} f-f^{\prime 2}+1\right) & =\frac{2}{3} S q-S \frac{\eta}{3} q^{\prime}+S \frac{G_{1}}{2} g-\frac{H\left(V^{2}\right)}{2}\left(f^{\prime \prime} V^{2}\right)^{\prime}, \\
q^{\prime} & =g, \\
\frac{1}{\sigma} g^{\prime \prime}-\frac{1}{3}\left(f^{\prime} g-2 f g^{\prime}\right) & =-\frac{\sigma}{2} H\left(V^{2}\right)\left(g^{\prime} V^{2}\right)^{\prime}
\end{aligned}
$$

and

$$
H\left(V^{2}\right)\left(g^{\prime}+\frac{1}{G r_{0} \sigma}\right)=0,
$$

with boundary conditions

$$
\begin{aligned}
& f=f^{\prime}=g-1=0, \quad \text { on } \eta=0, \\
& f^{\prime}-1=g=q=0, \quad \text { as } \eta \rightarrow \infty
\end{aligned}
$$

and the requirement that $f, f^{\prime}, f^{\prime \prime}, g, g^{\prime}$ and $q$ are continuous at $\eta=\eta_{2}$ where $V=0$.

The numerical solution of the above equations follows a similar procedure to that outlined for the solution of (22a) and (23). We now make initial guesses for $f^{\prime \prime}(0)$, $g^{\prime}(0), q(0)$ and $V(0)$ and integrate (27a) and (28) from $\eta=0$ to $\eta=\eta_{2}^{*}$, where $V\left(\eta_{2}^{*}\right)=0$ (to within some specified tolerance). Equation (27a) is then integrated from $\eta=\eta_{2}^{*}$ to $\eta=\eta_{\infty}$, with initial conditions obtained from the requirement of continuity of the dependent variables at the edge of the vortex region. Newton iteration is then used to update the unknowns at the wall until the far-field boundary conditions are satisfied.

In Figure 7 we present a plot of the extent of the region of vortex activity and the vortex induced skin-friction as a function of the curvature parameter for a variety of values of $S$. For a given value of $G_{1}>0$ (for the precise meaning of $G_{1}$ see the earlier discussion in Section 3) both the extent of the region of vortex activity and the vortex strength, as evidenced by the skin friction, are increased as $S$ increases. Such a simple conclusion cannot be drawn if $G_{1}<0$; indeed for $G_{1} \leq-2$ the converse holds. Furthermore, the skin friction induced by the vortex is, for a particular value of $S$, decreased over that found in the absence of the vortex.

In Figure 8 we again present a plot of the extent of the region of vortex activity, now as a function of $G r_{0}$, for a variety of values of $G_{1}$. The results in this figure demonstrate that the stabilizing effect of the retarding buoyancy/curvature induced acceleration, for which $G_{1}<0$, can be overcome by increasing the level of buoyancy through heating the fluid (that is, increasing $G r_{0}$ ).

Finally in Figure 9 we present a plot of the stream-wise velocity and the temperature for the case $S=1$ for a variety of values of $G_{1}$. Comparison with Figure 2 (the unperturbed boundary layer flow) clearly demonstrates that the nonlinear vortex structure slightly increases the value of the maximum of the stream-wise velocity. The 


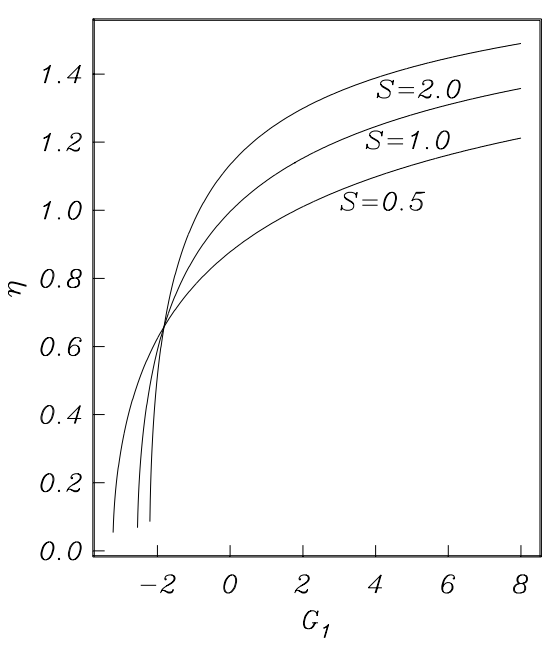

(a)

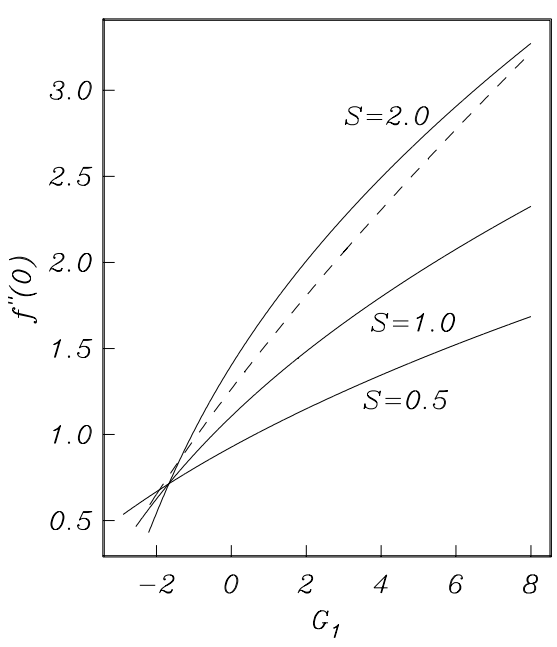

(b)

FigurE 7. (a) The range of existence of the vortex, (b) $f^{\prime \prime}(0)$ (skin friction), as functions of the scaled Görtler number with $\sigma=1, G r_{0}=2$ and $S=0.5,1,2$. The dashed curve in (b) is the skin friction for the basic boundary layer flow, in the absence of the vortex, for the case $S=1$.

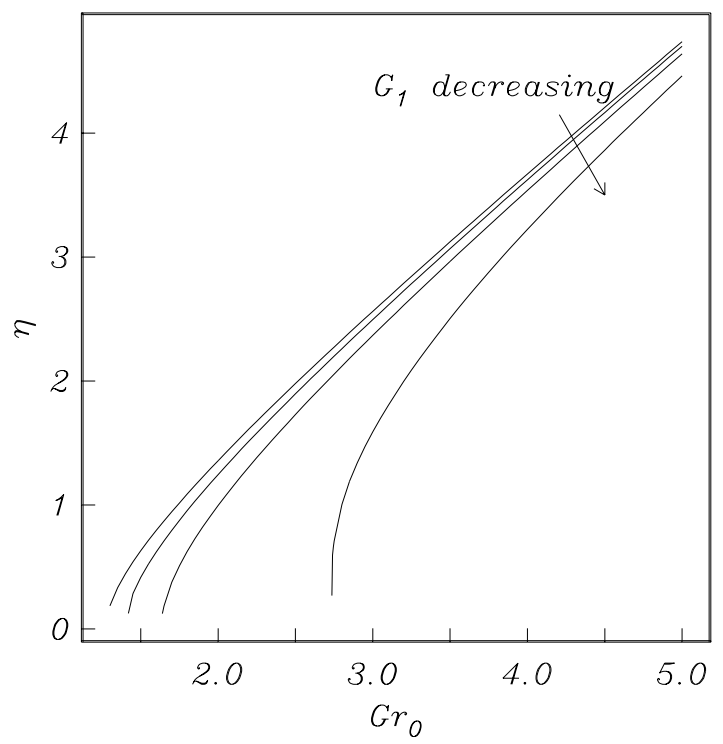

FIGURE 8. The range of existence of the vortex as a function of scaled Grashof number with $\sigma=1$, $G_{1}=8,4,0,-4$ and $S=1$. 


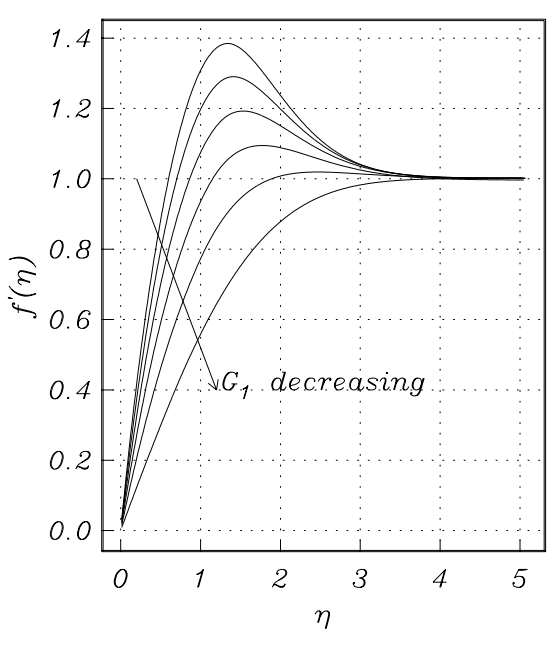

(a)

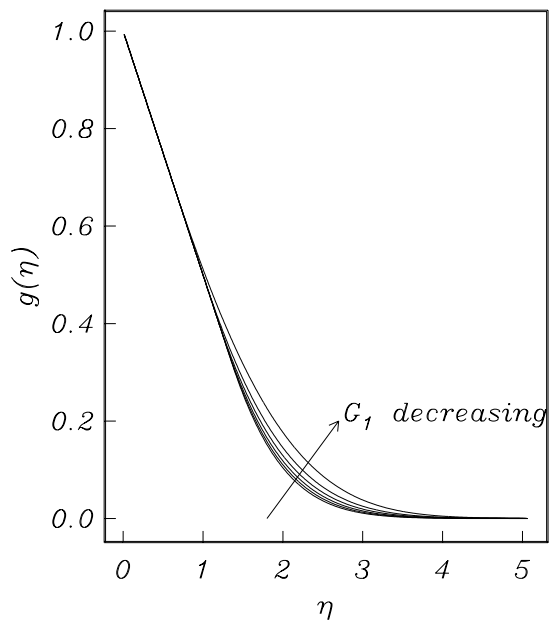

(b)

FIGURE 9. An eigenfunction plot for (a) $f^{\prime}\left(\eta\right.$ ) versus $\eta$, (b) $g\left(\eta\right.$ ) versus $\eta$ for $G_{1}=-2,0,2,4,6,8$ with $S=1$ and $\sigma=1$.

nonlinear vortex thus acts to enhance the accelerating effect of the thermal buoyancy within the flow.

\section{Conclusions}

In this paper we have considered the instability of buoyancy driven boundary layers to linear and nonlinear vortex disturbances in the naturally occurring limit of large Grashof number. Attention has focused on the question of how centrifugal forces, present when the heated surface is curved, serve to modify the stability properties of the flow. It has been shown that both the linear and nonlinear stability characteristics are dependent on whether the buoyancy parameter $S=G r / R e$ can be considered negligible or not.

In Section 3 it was shown in the case of linear inviscid disturbances, if we assume $S$ to be negligible, then the effects of curvature first become significant when the Grashof number and Görtler number are of the same size. In this situation it was found that the introduction of a positive curvature across a plate with a positive temperature gradient increases the growth-rates of already unstable modes. If the plate is curved in a convex manner the flow was found to be unstable with the growth-rates of the disturbances decreasing with increasing negative Görtler number. However, no unstable modes were found for stably stratified flows (corresponding to $G r<0$ ). If we consider the buoyancy parameter $S$ to be non-negligible, it is shown that the effects of curvature are felt in the basic flow when the local slope of the body, and hence 
the local Görtler number, is an order one quantity. The effect of curvature is felt, not through a centrifugal acceleration term in the disturbance equations but instead through a buoyancy/curvature induced acceleration in the underlying basic flow. The fastest growing modes are modified through the introduction of the competing sources of instability, namely buoyancy and stream-wise curvature. The growth-rates of these, the dominant mode of disturbance, increase with increasing Görtler number and decrease as the level of convex curvature increases. For a given level of buoyancy, parametrised by $S$, all modes were found to be stabilised at sufficiently large negative Görtler numbers.

The nonlinear development of short-wavelength vortices was considered in Section 4. In the combined limit of large Grashof and Görtler numbers the effect of buoyancy is to enhance the nonlinear vortex motion, and as such, allow steady, spanwise periodic, vortices to persist into parameter régimes where the curvature induced centrifugal acceleration of the flow is not sufficient to support such motions. Furthermore, in the case when buoyancy coupling is significant, that is, when $S \neq 0$, the effect of buoyancy can overcome the stabilizing influence of convex curvature. The nonlinear vortex state can also act to accelerate the boundary layer thus increasing the maximum value of the stream-wise velocity.

Recently, a novel wave/mean flow interaction theory (not unlike that encountered in the present work) has been presented by Denier and Mureithi [6] and Denier and Stott [7] for strongly buoyant boundary-layer flows whose stream-wise velocity component attains a maximum. In such a theory the nonlinear wave travels at a speed equal to the maximum of the unperturbed, stream-wise velocity, has a small wavelength in the stream-wise direction and induces a mean flow correction of a size comparable to the wave amplitude. The fact that such a maximum velocity persists, and is enhanced, in the presence of the nonlinear vortex state suggests that the mechanism by which the flow can support the nonlinear travelling waves of [6] and [7] will be operable. The possibility of interactions between the short wavelength longitudinal vortices and short wavelength travelling waves then arises. Also as noted earlier the nonlinear vortex states described in Section 4 are susceptible to secondary instabilities, in this case in the form of Rayleigh waves. These are a manifestation of the varicose and sinuous time dependent motions that are observed experimentally in Görtler vortex flows. How these waves interact with the purely buoyancy driven short waves described in Denier and Mureithi [6] presents an interesting and challenging problem. We hope to be able to report on the solution of this problem in the near future.

\section{Acknowledgements}

Thanks to Drs Stephen Cowley and Stephen Otto, as well as an anonymous referee, whose comments helped to greatly improve the presentation of this paper. 


\section{References}

[1] M. Abramowitz and I. A. Stegun, Handbook of mathematical functions (Dover, New York, 1965).

[2] G. K. Batchelor, An introduction to fluid dynamics (Cambridge University Press, London, 1967).

[3] N. D. Blackaby and M. Choudhari, "Inviscid vortex motions in weakly three-dimensional boundary layers and their relation with instabilities in stratified shear flows", Proc. R. Soc. Lond. (A) 440 (1993) 701-710.

[4] J. P. Denier and P. Hall, "Fully nonlinear Görtler vortices in constricted channel flows and their effect on the onset of separation", NASA/ICASE contractor report 92-29.

[5] J. P. Denier, P. Hall and S. O. Seddougui, "On the receptivity problem for Görtler vortices: vortex motions induced by wall roughness", Phil. Trans. R. Soc. Lond. (A) 335 (1991) 51-85.

[6] J. P. Denier and E. W. Mureithi, "Weakly nonlinear wave motions in a thermally stratified boundary layer", J. Fluid Mech. 315 (1996) 293-316.

[7] J. P. Denier and J. A. K. Stott, "Wave-mean flow interactions in thermally stratified Poiseuille flow”, Stud. Appl. Math. 102 (1999) 121-136.

[8] P. G. Drazin and W. H. Reid, Hydrodynamic stability (Cambridge University Press, London, 1981).

[9] J. L. Goldstein and E. M. Sparrow, "Characteristics for flow in a corrugated wall channel", J. Heat Transfer 99 (1977) 187-195.

[10] P. Gschwind, A. Regele and V. Kottke, "Sinusoidal wavy channels with Taylor-Görtler vortices", Exp. Thermal Fluid Sci. 11 (1995) 270-275.

[11] P. Hall, "The linear development of Görtler vortices in growing boundary layers", J. Fluid Mech. 130 (1983) 41-58.

[12] P. Hall, "Streamwise vortices in heated boundary layers", J. Fluid Mech. 252 (1993) 301-324.

[13] P. Hall and N. J. Horseman, "The inviscid secondary instability of fully nonlinear longitudinal vortex structures in growing boundary layers", J. Fluid Mech. 232 (1991) 357-375.

[14] P. Hall and W. D. Lakin, "The fully nonlinear development of Görtler vortices in growing boundary layers", Proc. R. Soc. Lond. (A) 415 (1988) 421-444.

[15] P. Hall and H. Morris, "On the instability of boundary layers on heated flat plates", J. Fluid Mech. 245 (1992) 367-400.

[16] P. Hall and S. O. Seddougui, "On the onset of three-dimensionality and time-dependence in Görtler vortices", J. Fluid Mech. 204 (1989) 405-420.

[17] A. M. Jacobi and K. S. Ramesh, "Air-sided flow and heat transfer in compact heat exchangers: a discussion of enhancement mechanisms", Heat Trans. Eng. 19 (1998) 29-41.

[18] S. M. Mangalam, J. R. Dagenhart, T. E. Hepner and J. F. Meyers, "The Görtler instability on an airfoil", AIAA Paper 85-0491, 1985.

[19] E. W. Mureithi, "Effects of thermal buoyancy on the stability properties of boundary layer flows", Ph. D. Thesis, University of New South Wales, 1998.

[20] E. W. Mureithi, J. P. Denier and J. A. K. Stott, "The effect of buoyancy on upper branch TollmienSchlichting waves”, IMA J. App. Math. 58 (1997) 19-50.

[21] S. N. Timoshin, "Asymptotic analysis of a spatially unstable Görtler vortex spectrum", Fluid Dyn. 25 (1990) 25-33.

[22] G. Wang and P. Vanka, "Convective heat transfer in periodic wavy passages", Int. J. Heat Mass Trans. 38 (1995) 3219-3230. 